\title{
3 Research Square \\ Effects of Voids and Raster Orientations on Fatigue Life of Notched Additively Manufactured PLA Components
}

Soran Hassani Fard ( $\sim$ s.hassanifard@mail.utoronto.ca )

University of Toronto

Kamran Behdinan

University of Toronto

\section{Research Article}

Keywords: Additive Manufacturing, Fatigue life, Notch sensitivity, Defects

Posted Date: February 7th, 2022

DOI: https://doi.org/10.21203/rs.3.rs-1310180/v1

License: (c) (i) This work is licensed under a Creative Commons Attribution 4.0 International License.

Read Full License 


\title{
Effects of voids and raster orientations on fatigue life of notched additively manufactured PLA components
}

\author{
Soran Hassanifard*, and Kamran Behdinan \\ Advanced Research Laboratory for Multifunctional Lightweight Structures (ARL-MLS), Department of \\ Mechanical and Industrial Engineering, University of Toronto, Toronto, Canada \\ Corresponding Author: (Email: s.hassanifard@mail.utoronto.ca)
}

\begin{abstract}
In this study, the fatigue life of notched Polylactic acid (PLA) samples fabricated through the fused deposition modeling (FDM) technique was studied experimentally and numerically. The volumetric method based on the theory of critical distance was employed for fatigue life predictions. The effects of influential process parameters including raster orientation and FDMinduced defects such as voids or gaps inside the parts were examined on the fatigue strength reduction factors and fatigue lives. Circular and elliptical-shaped notch geometries were considered with various dimensions. Fatigue tests were conducted on notched and un-notched samples at the load ratio of 0.1 . Predicted results were compared with experimental fatigue test data. Results revealed that the raster orientation parameter had a substantial impact on fatigue strength reduction factors and fatigue lives. The stress concentrations induced by the FDM process on the surfaces and inside the parts for the samples with $90^{\circ}$ raster angles acted similar to the sharp notches, resulting in no substantial difference in fatigue life of notched and un-notched 3D printed samples. In contrast, un-notched $3 \mathrm{D}$ printed specimens with $0^{\circ}$ raster orientations possessed higher fatigue lives as compared to the notched samples. While the volumetric approach efficiently predicted the fatigue lives of the samples with $90^{\circ}$ raster orientations, it moderately underpredicted the fatigue lives of the samples with $0^{\circ}$ raster angles.
\end{abstract}

Keywords: Additive Manufacturing, Fatigue life, Notch sensitivity, Defects. 


\section{Nomenclature:}

\begin{tabular}{|ll|ll|}
\hline \multicolumn{2}{|l|}{ List of symbols and acronyms: } & & \\
& & \\
$\sigma_{f}^{\prime}:$ & Fatigue strength coefficient & $a$ and $b:$ & Large and small ellipse diameters \\
$\varepsilon_{f}^{\prime}:$ & Fatigue ductility coefficient & $X_{e f f}:$ & Effective distance \\
$b:$ & Fatigue strength exponent & $\chi:$ & Stress gradient \\
$c:$ & Fatigue ductility exponent & R: & Stress ratio \\
$N_{f}:$ & Fatigue life cycles & $\sigma_{a}:$ & Stress amplitude \\
$E:$ & Elastic modulus & $\varepsilon_{a}:$ & Strain amplitude \\
$\sigma_{\text {max }}:$ & Maximum stress & $K_{f}:$ & Fatigue notch factor \\
$\sigma_{y}:$ & Yield strength & $K_{t}:$ & Elastic stress concentration factor \\
$\alpha:$ & Peterson's constant & AM: & Additive manufacturing \\
$\beta:$ & Neuber's constant & FDM: & Fused deposition modeling \\
$\rho:$ & Notch tip radius & PLA: & Polylactic acid \\
$q:$ & Notch sensitivity & UTS: & Ultimate tensile strength \\
& & TCD: & Theory of critical distance \\
\hline
\end{tabular}

\section{1- Introduction}

With the development of Additive Manufacturing (AM) techniques, also known as three dimensional (3D) printing, the need for fabricating components with complex geometries are increasing. Several AM techniques have been developed for manufacturing metallic and polymeric parts [1]. The most commonly used material extrusion-based printing method for fabricating plastic components is Fused Deposition Modelling (FDM). This technique has gained extensive popularity due to its simplicity, broad filament availability, and low cost $[2,3]$. In this technique the raw plastic material passes through a nozzle, then the filament is deposited over the previously laid layer of the part to form a desired shape [3].

During the FDM process, filament temperature reaches to the melting point to help the filaments stick easily to each other, followed by a relatively rapid cooling. Rapid solidification may cause residual stresses in the part, and often results in forming micro-cracks, distortion, deformation, and other types of defects [4]. Missing filament materials during the FDM process, and air gaps or voids after the process are other features of defects in FDM-produced polymeric parts as compared to the conventionally manufactured plastic components. Defects such as voids or air gaps as well as rough surfaces of the parts can adversely affect the mechanical performance of the components [5-7]. Fayazbakhsh et al. showed that defects in the form of missing extrudates during the FDM process reduced the tensile strength of the AM PLA parts significantly [8]. Kerekes et al. [9] studied the damage and deformation of FDM-processed AM parts through conducting several tensile tests and employing inverse identification analysis. They observed that the area with premature pores in the intersection of infill and filaments outline shell was the region where failures initiated from. In the FDM process, depending on the nuzzle diameter while fabricating the parts, surface roughness as well as the percentage of the voids or gaps inside the part can vary. Surface roughness and the stress concentrations induced by the intrinsic voids in the FDMprocessed parts can have detrimental effects on the mechanical properties of the AM parts. 
The orientation of the filament deposition during the process as well as the part build direction can also have major impact on mechanical characteristics of the AM parts [10, 11]. It has been observed through various research studies that different AM plastic materials undergoing static or cyclic loadings behave differently with respect to the raster orientation. Ziemian et al. [12] reported anisotropic effects in both tensile strength and fatigue performance of acrylonitrile butadiene styrene 3D-printed parts. The showed that fatigue life of the samples in longitudinal $\left(0^{\circ}\right)$ and default $\left(45 / 45^{\circ}\right)$ raster orientations was noticeably higher than that of diagonal $\left(45^{\circ}\right)$ or transverse $\left(90^{\circ}\right)$ orientations. Afrose et al. [13] studied the influence of raster orientation on fatigue performance of PLA 3D-printed components. They observed that the parts in $45^{\circ}$ build orientation possessed higher fatigue life than those fabricated with $0^{\circ}$ and $90^{\circ}$ raster angle while the samples were undergoing the same percentage of applied static loads. FDM manufacturing technique have other process parameters which can have significant impact on static and fatigue strength of the AM parts.

Most of the components in service contain at least one feature of geometrical discontinuities including key holes, notches, grooves, and so on. These geometrical discontinuities adversely affect the performance of the parts being subjected to various load conditions including static, dynamic, impact, or cyclic loads. Due to distinct manufacturing characterizations, 3D printed parts behave differently as compared to those parts manufactured through traditional techniques such as casting or injection molding. It is well known that existing a notch in a component can dramatically reduce its fatigue strength. A number of research studies can be found in the literature dealing with the effects of notches on static and fatigue performance of AM metallic parts [14-18] and polymeric components [19-22]. However, since the nature of 3D printed metallic and polymeric parts fabricated through specific AM techniques is totally different, these components behave differently with respect to the notches as well. The Murakami model based on the $\sqrt{\text { area }}$-approach was successfully employed for predicting fatigue life of notched and un-notched Inconel 718 AM samples [23, 24]. Molaei and Fatemi [25] studied fatigue characteristics of notched Ti-6Al-4V and 17-4 PH stainless steel additively manufactured samples undergoing axial and combined axialtorsion variable amplitude loads. They reported that due to rough surface of the as-built sample, both notched and un-notched specimens could be treated as notched sample having different values of fatigue notch factors.

There is a lack of sufficient understanding of the effects of FDM-related voids or residual gaps on the overall mechanical performance of the AM plastic parts. Taken a step further, the information about the influence of these voids or residual gaps on the fatigue strength of the plastic parts being manufactured using the FDM technique is scarce. Due to the complexity of the nature of the FDM process and involving many influential process parameters including anisotropy, surface roughness, and voids/gaps, further investigations are required to fully understand fatigue characteristics of the notched FDM-processed plastic samples. In this study, the focus is placed on the influence of the raster orientation factor and its effects on fatigue life of notched PLA samples fabricated through the FDM technique, taking into account the effects of intrinsic voids or residual air gaps in the samples. Cycle-dependent fatigue strength reductions factors $K_{f}^{\prime}(N)$ are employed to predict the fatigue life of notched samples based on a Theory of Critical Distance (TCD) and using the available fatigue reference curve of the smooth neat PLA material. In the end, predicted 
fatigue lives using this approach are compared with those obtained via experiments, and the discrepancies in the results have been interpreted based on the manner of the failures in the specimens.

\section{2- Theoretical approaches \\ 2-1 Notch effects}

The S-N curve of notched components can be predicted using the S-N curve of un-notched samples. It can be considered that fatigue strength of un-notched samples at a certain life is $K_{t}$ times greater than that of notched specimen, where $K_{t}$ is the elastic stress concentration factor. This is simplest and most conservative method of fatigue life prediction of notched samples. Because, it is well known that fatigue notch factor $\left(K_{f}\right)$, which is defined as the ratio of smooth fatigue limit to the notched fatigue limit, is always smaller than the elastic stress concentration factor. One reason for this fact is that the stresses in the notched samples exceed the elastic limit even at low load levels, resulting in expanding plastic strain zone in the vicinity of the notch instead of increasing stress value. Fatigue notch factor can also be expressed based on notch sensitivity (q) as follows [26]:

$q=\frac{K_{f}-1}{K_{t}-1} \quad 0 \leq q \leq 1$

For an elastic-perfectly plastic model, three different values for $K_{f}$ can be determined based on the applied load level and yielding condition. For the cases of respectively (i) no yielding, (ii) local yielding, and (iii) full yielding (completely reversed), $K_{f}=K_{t}, K_{f}=\frac{\sigma_{y}}{s_{a}}$, and $K_{f}=1$ [26]. In this equation, $S_{a}$ is the stress amplitude, and $\sigma_{y}$ is yield stress. However, for a wide variety of materials the elastic-perfectly plastic model is not a suitable plasticity model and leads to erroneous results.

\section{2-2 strain-based approaches to fatigue}

In this method, total strain component which is the summation of elastic and plastic strain values is related to the number of cycles to failure. There are an extensive number of strain-based models and criteria which have been proposed to predict fatigue life. The conventional Smith-WatsonTopper (SWT) model [27] is one of the most commonly used strain-based approaches that can be used for fatigue life estimations of wide variety of materials. The SWT model can be expressed as follows:

$\varepsilon_{a} \sigma_{\max }=\frac{\left(\sigma_{f}^{\prime}\right)^{2}}{E}\left(2 N_{f}\right)^{2 b}+\sigma_{f}^{\prime} \varepsilon_{f}^{\prime}\left(2 N_{f}\right)^{b+c}$

In this equation $\sigma_{f}^{\prime}, \mathrm{b}, \varepsilon_{f}^{\prime}$, and $\mathrm{c}$ are strain-based fatigue parameters related to both elastic and plastic lines of the $\varepsilon-N$ curve. In order to use strain-based approaches such as SWT model to predict fatigue life, the above-mentioned fatigue parameters should be known. These parameters can be found in the literature for a wide variety of metals and alloys such as steels and aluminum 
alloys, however, they can rarely be found for AM materials due to the manufacturing-related complexity and anisotropy of these components. Some strain-based fatigue parameters for a number of AM materials can be found in reference [28]. However, these parameters are limited for certain conditions and cannot be generalized.

\section{2-3 Theory of critical distance}

The most basic concepts on what is known as the theory of critical distance were proposed by Neuber [29] and Peterson [30]. Neuber assumed that the mean stress over a certain structural size which is also known as "critical distance" ahead of the notch could be used for evaluating notch sensitivity [29]. Based on the Neuber's relation, notch sensitivity can be obtained as follows:

$q=\frac{1}{1+\sqrt{\beta / \rho}}$

where $\beta$ is a material constant that can be determined through experiments, and $\rho$ is the radius of the notch root. Peterson assumed that fatigue failure occurs when the stress at a certain point ahead of the notch root reaches to the fatigue strength of the un-notched component [30]. Based on the Peterson's assumption of reducing stress in the vicinity of the notch tip linearly, the notch sensitivity can be expressed as follows:

$q=\frac{1}{1+\alpha / \rho}$

In Peterson's relation $\alpha$ is a material constant and should be obtained experimentally. Although for some materials such as steels or aluminum alloys there can be found some empirically obtained material constants of Peterson's and Neuber's models, for other materials these constants are not available and need to be determined through experiments. These material constants are not available for AM plastic materials, as well. Similar to the models proposed by Neuber and Peterson, there are other methods that can determine critical length parameters for cracks and can be adopted for assessing notch fatigue limits [31, 32]. Research studies on fatigue assessments of AM materials based on the TCD theory are very limited $[22,25]$.

\section{2-4 Volumetric approach}

To have a more precise fatigue life prediction of notched AM samples especially for notchsensitive materials such as PLA, it is necessary to utilize a method which does not require complex constitutive models, and can be implemented for notches with different geometrical features. Volumetric approach which has been proposed by Pluvinage [33], is based on the theory of critical distance approach and can be considered as a powerful candidate for fatigue life prediction of the notched components. In this approach, determining the magnitudes of effective stress and the effective distance is of great importance. The effective distance is a length measured from the notch root at which the fatigue phenomenon is expected to occur. The effective stress is defined as the average of the weighted stresses over effective distance [33]. 
Figure 1 shows a typical elastic-plastic stress distribution and stress gradient ahead of a typical notch in a notched specimen. The relative stress gradient can be defined as follows:

$\chi=\frac{1}{\sigma(x)} \frac{d \sigma(x)}{d x}$

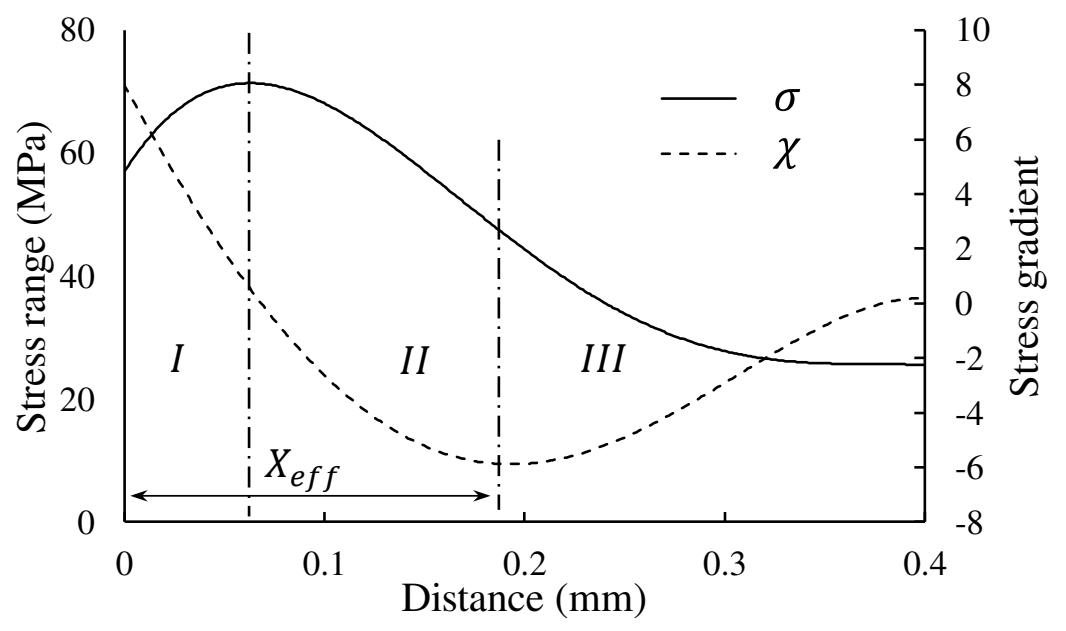

Fig. 1. Elastic-plastic stress distribution in the vicinity of a typical notch [33]

Three distinct zones in Figure 1 are recognized each having important characteristics. These zones are named as zones I, II, and III. Zone I corresponds to a distance calculated from the notch root to the point with maximum stress value. The effective distance has been found to be the limit between zones II and III. For the cases where plastic strain components do not exist, the stress value at the notch tip is the maximum, and zone I vanish. According to this method, fatigue notch factor can be obtained using the following formula:

$K_{f}=\frac{1}{X_{e f f} \sigma_{n}} \int_{0}^{X_{e f f}} \sigma_{y y}(x)(1-x \chi) d x$

In this equation, $X_{e f f}$ is the effective distance, $\sigma_{n}$ is the net stress, $\sigma_{y y}$ is the stress along the loading direction. After obtaining fatigue notch factors, the reference fatigue curve of smooth specimens can be utilized for fatigue life prediction of the notched samples.

\section{3- Experiments}

\section{3-1 Materials and specimens}

The AM samples were fabricated at $0^{\circ}$ and $90^{\circ}$ raster orientations. The nozzle diameter of conventional FDM 3D printer devices vary usually between $0.2 \mathrm{~mm}$ to $0.8 \mathrm{~mm}$. This parameter impacts the quality and overall mechanical properties of the plastic specimens being 3D printed. In this research, the largest possible nozzle diameter $(\mathrm{D}=0.8 \mathrm{~mm})$ was chosen in order to highlight and to have a better understanding of the effects of residual gaps or voids on fatigue performance of 3D printed notched samples. Also, three different notch shapes were selected, one circular 
shaped with $r=2 \mathrm{~mm}$, and two elliptical shaped with b/a ratio of respectively 0.5 and 0.25 ( $a=4 \mathrm{~mm}$ is the large diameter and $b$ is the small diameter of the ellipse). Figure 2 shows the schematic views of the samples at $0^{\circ}$ and $90^{\circ}$ raster orientations as well as the geometry of the samples and notches.
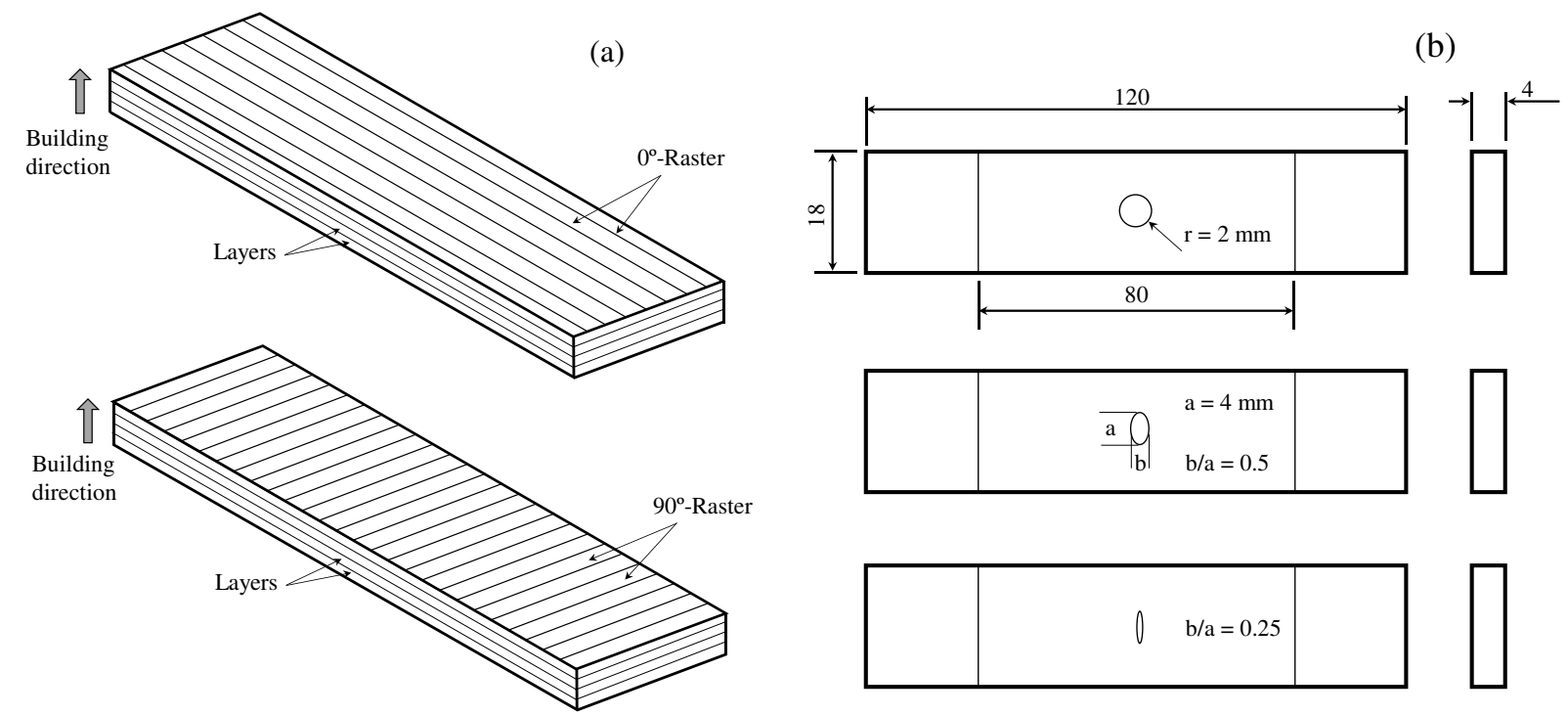

Fig. 2. The schematic view of the 3D printed samples; (a) building direction and raster angles, (b) geometries of the notched specimens.

\section{3-2 Static tests of un-notched samples}

Quasi-static tests of 3D printed samples were conducted to obtain the stress-strain curves of the un-notched samples. Three tests according to ASTM D638 standard were carried out for each condition and the average value of the Ultimate Tensile Strength (UTS) was calculated. Figure 3 illustrates the stress-strain curves of un-notched samples at $0^{\circ}$ and $90^{\circ}$ raster orientations. The tensile strength of the PLA filament or that of injection molded could be quite different with the strength of 3D printed parts [13]. In this study, the static and fatigue properties of pure PLA material are used for respectively (i) FE simulations to obtain stress distributions, and (ii) fatigue life predictions based on the volumetric approach. Therefore, the stress-strain curve of a generic pure PLA material available in ref. [34] was included in Figure 3. The ultimate tensile strength of the $3 \mathrm{D}$ printed sample at $0^{\circ}$ raster angle was $68 \%$ of the neat PLA material. Afrose et al. [13] reported that this ratio could roughly vary between 60 to $64 \%$. 


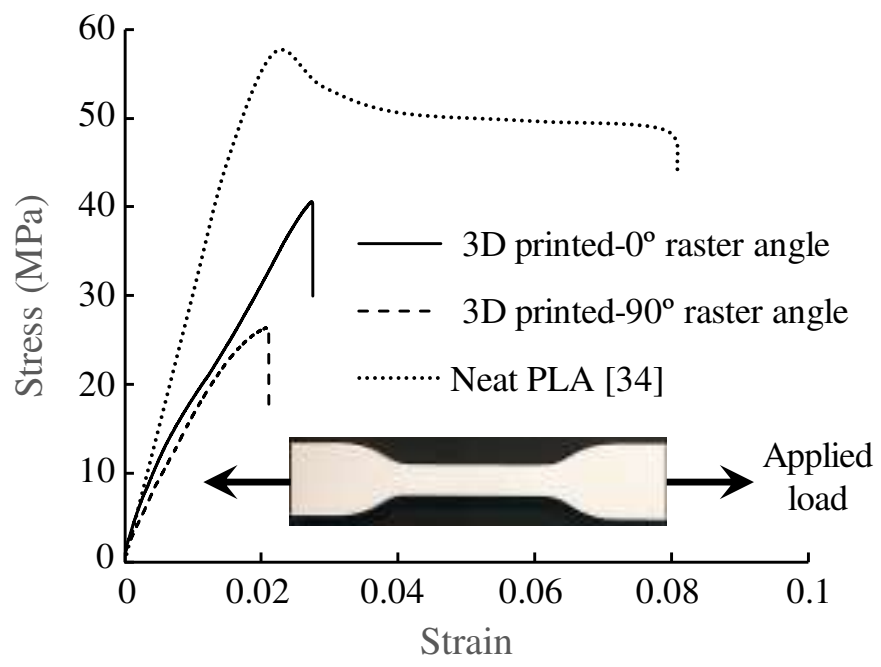

Fig. 3. Stress-strain curves of the un-notched $A M$ samples at $0^{\circ}$ and $90^{\circ}$ raster orientation as well as that of neat PLA material

\section{3-3 Fatigue tests of smooth and notched samples}

Fatigue tests were carried out using Instron 5900 series fatigue testing machine at frequency of 0.5 $\mathrm{Hz}$ and load ratio of $\mathrm{R}=0.1$. Cyclic loads were selected based on certain percentages of the UTS values of the samples. Experimental fatigue test data is the average of three tested specimens at each load level. Figure 4 shows the S-N curves of un-notched and notched samples as well as the reference fatigue curve of PLA material. The reference curve, which will be used to predict fatigue life of notched samples, were plotted based on the fatigue test data for the neat PLA samples available in ref. [34]. Since the fatigue test data of 3D printed samples and that of reference fatigue curve are in different load ratio, both sets of fatigue test data were converted to the completely reversed condition loading $(\mathrm{R}=-1)$ using the $\mathrm{SWT}$ parameter $\sigma_{a r}=\sqrt{\sigma_{\max } \sigma_{a}}$ [27].

(a)

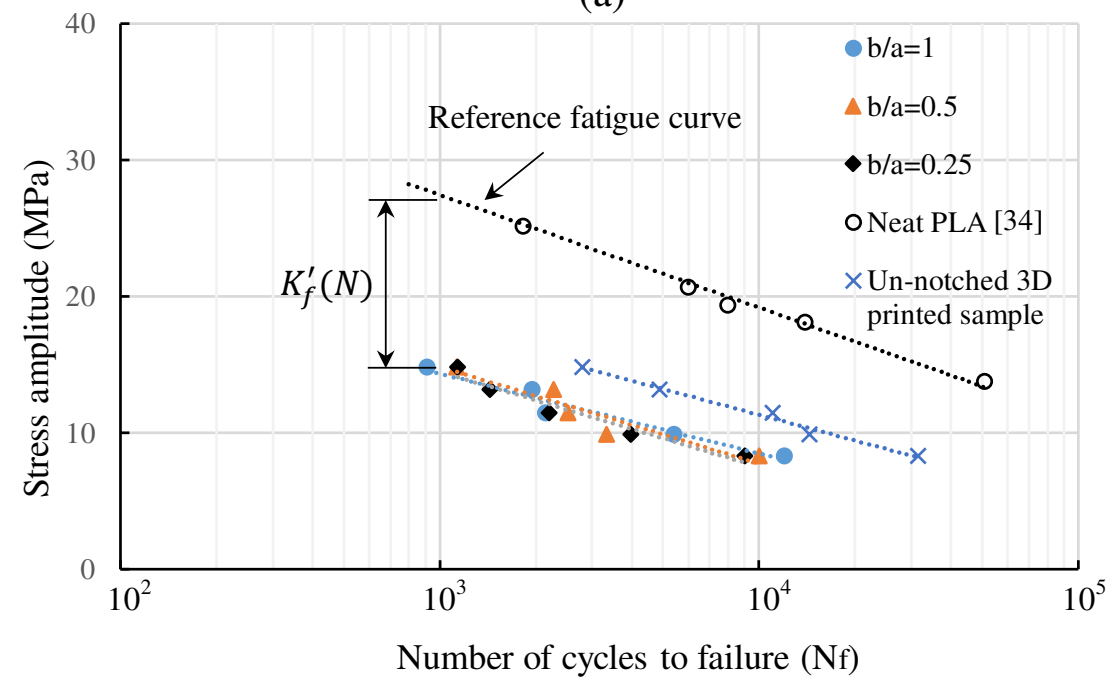


(b)

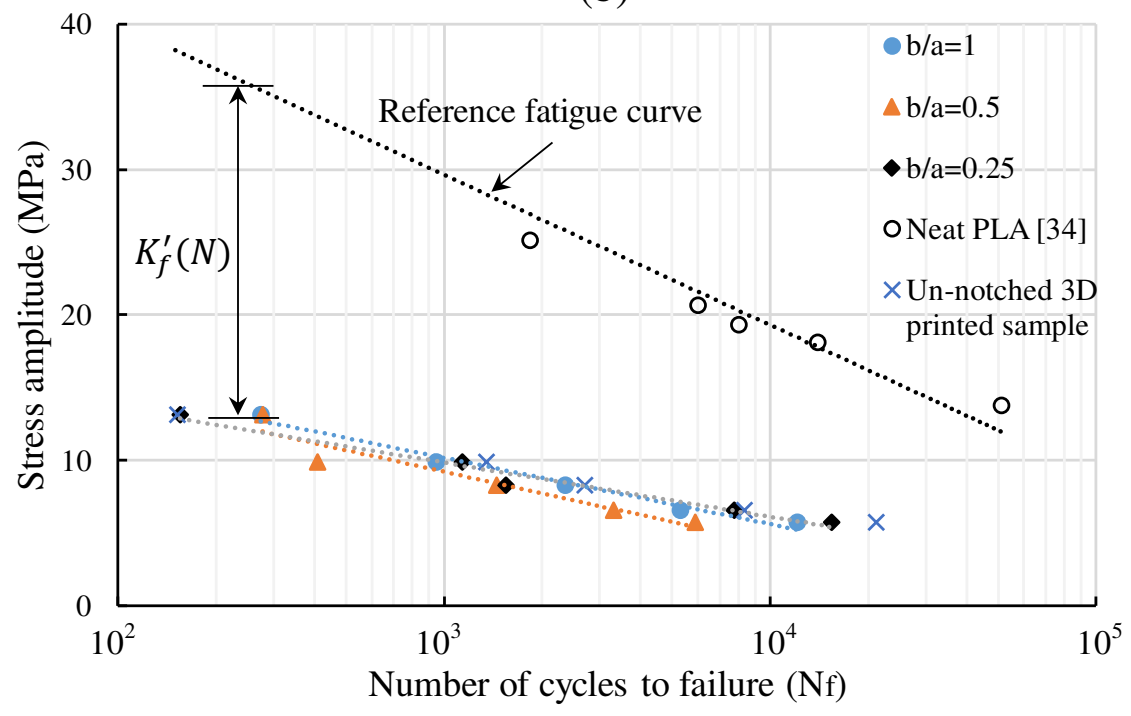

Fig. 4. Fatigue test data of notched and un-notched $A M$ samples at (a) $0^{\circ}$, and (b) $90^{\circ}$ raster orientation. (The reference fatigue curve for the neat PLA has been extracted and reproduced based on the data available in reference [34])

The S-N curves of the samples with $90^{\circ}$ raster orientation clearly indicate that the impact of stress concentration caused by the FDM process was greater than that of notches, as evidenced in Figure 4(b). In fact, 3D printed un-notched samples with $90^{\circ}$ raster orientation behave similarly to the notched samples. This is mainly due to poor bonding between the filaments where the load tends to separate filaments from each other. In such case, micro cracks initiated in the vicinity of the notch holes where the filaments poorly bonded to each other and then propagated very rapidly through the interface of the filaments, as evidenced schematically in Fig. 5(a). A crack initiation site as well as its propagation path is shown in Fig. 5(b), indicating that the effects of intrinsic cracks and discontinuities were more prominent than that of notches. This effect was observed to be more dominant in low cycle fatigue regime. This was not the case for the samples with $0^{\circ}$ raster orientation. Fatigue strength of un-notched samples at $0^{\circ}$ raster angle was noticeably higher than those of notched specimens (see Figure 4(a)). For the case of samples with $0^{\circ}$ raster angle, cracks need to propagate along a line perpendicular to the filaments direction, resulting in an increased number of cycles to failure as compared to the samples with $90^{\circ}$ raster orientations. 
(a)

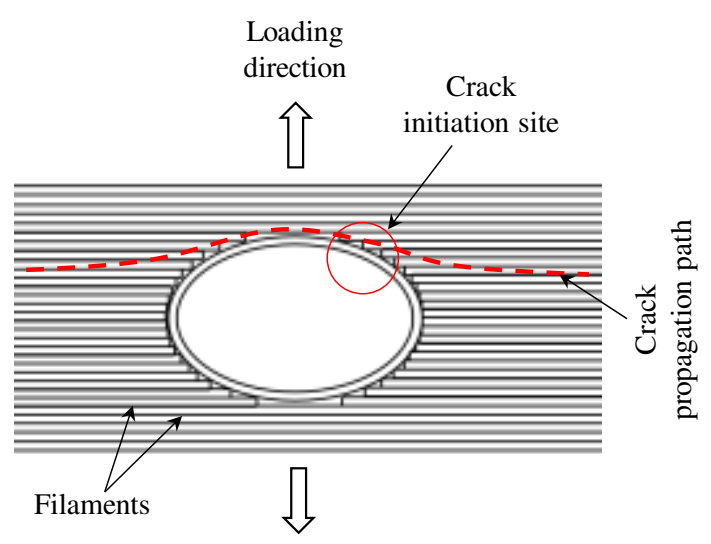

(b)

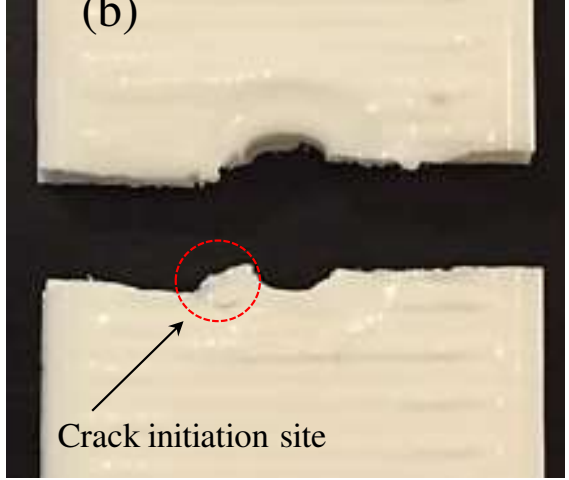

Fig. 5. Crack initiation and propagation mechanism in notched samples with $90^{\circ}$ raster angle, (a) schematic view, (b) actual fractured specimen

Since 3D printed PLA material acts similar to brittle materials, the components of plastic strains compared to the elastic ones can be negligible. Therefore, the S-N curves of the un-notched PLA samples can be used for determining fatigue strength coefficient and fatigue strength exponent based on the Coffin-Manson relation [26]:

$\sigma_{a r}=\sigma_{f}^{\prime}\left(2 N_{f}\right)^{b}$

In this equation, $\sigma_{a r}$ is the stress amplitude for the completely reversed loading condition. The calculated fatigue parameters based on the experimental fatigue test data of the un-notched specimens are summarized in Table 1.

Table 1. Fatigue parameters of 3D printed PLA parts at $0^{\circ}$ and $90^{\circ}$ raster orientations

\begin{tabular}{ccc}
\hline Raster angle & $\sigma_{f}^{\prime}(\mathrm{MPa})$ & $\mathrm{b}$ \\
\hline $0^{\circ}$ & 102.6 & -0.242 \\
$90^{\circ}$ & 32.5 & -0.174 \\
\hline
\end{tabular}

The data presented in Table 1 can be implemented for fatigue life prediction purpose for any notched samples at $0^{\circ}$ and $90^{\circ}$ raster angles. However, the maximum stress at the notch root could be noticeably greater than the effective stress value at which fatigue occurs. Therefore, to obtain more accurate results, the effective stress at the effective distance should be used for fatigue life predictions.

\section{4- FE simulations}

During the FDM process, the filaments are deformed and some gaps or voids are remained between the filaments, resulting in producing a part with a density lower than $100 \%$. This phenomenon is shown schematically in Fig. 6(a). The gaps have been observed in actual PLA specimens using 
Scanning Electron Microscopy (SEM) technique, as shown in Fig. 6(b). It is well known that most FDM-processed AM components show large level of anisotropy, however, in numerical simulations these parts may be treated as isotropic materials. In this study, the gaps between the filaments are simulated in FE analyses to obtain more accurate results.
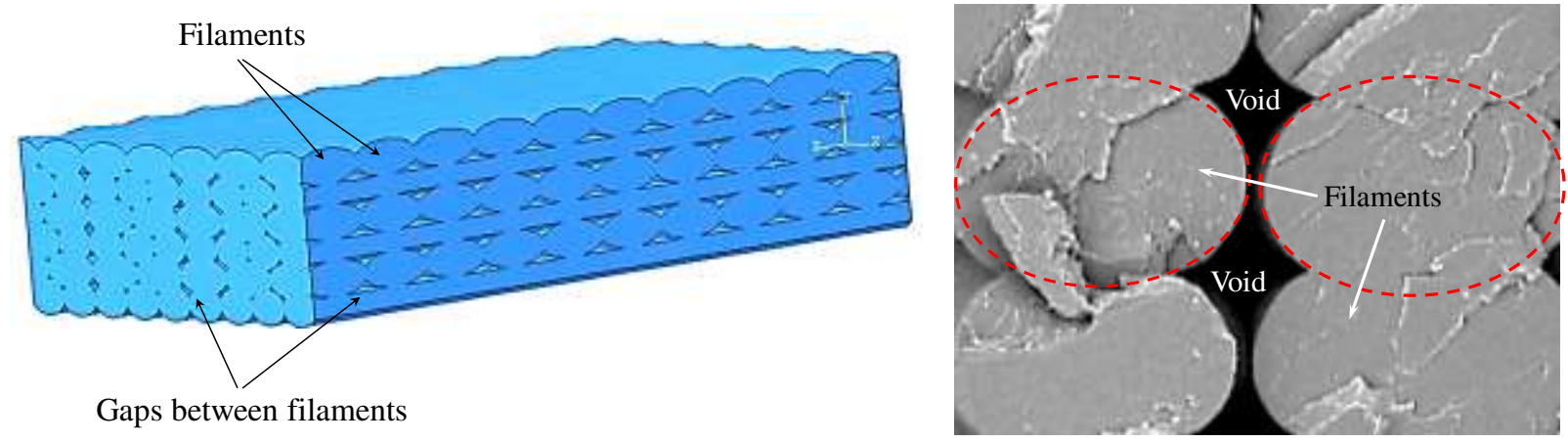

Fig. 6. Gaps between filaments in a typical FDM-produced sample; (a) FE model, and (b) SEM image of an actual specimen.

Figure 7 shows the way samples with $0^{\circ}$ and $90^{\circ}$ raster orientations were modeled in $\mathrm{FE}$ simulations. In Figure 7(a) filaments are parallel to the loading direction, while in Figure 7(b) the load is applied perpendicular to the filaments direction. The numerical simulations were performed using Abaqus software to obtain stress distributions along the lines starting from the notch tip toward outside of the notched samples.

(a)

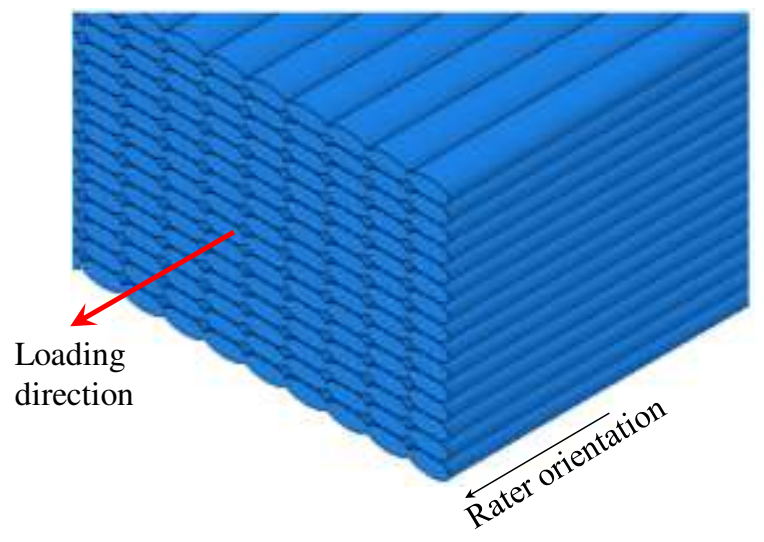

(b)

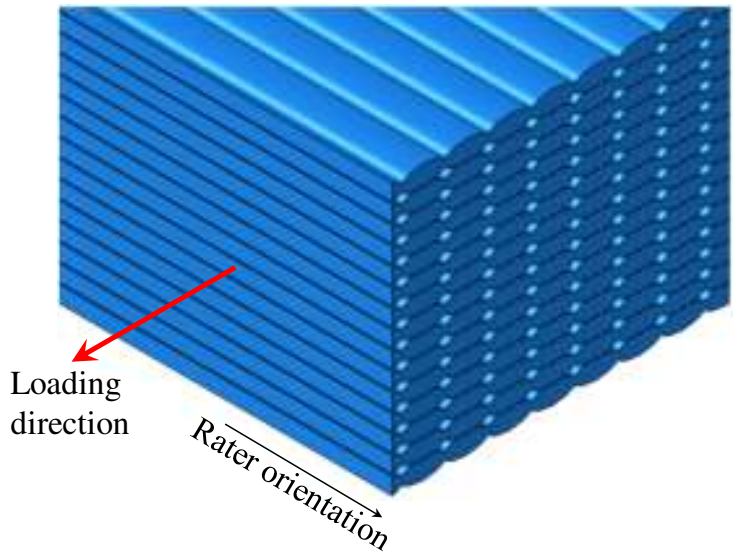

Fig. 7. Typical FE models of the samples with (a) $0^{\circ}$, and (b) $90^{\circ}$ raster orientations taking into account the effects of voids and gaps between the filaments 


\section{5- Results and discussions}

Figure 8 illustrates the stress distributions and relative stress gradients for notched samples at $0^{\circ}$ raster orientation undergoing the same nominal stress of 12.3 MPa. Similar graphs have been plotted for samples with $90^{\circ}$ raster orientation at all load levels ranging roughly between 50-80 percent of the UTS values.
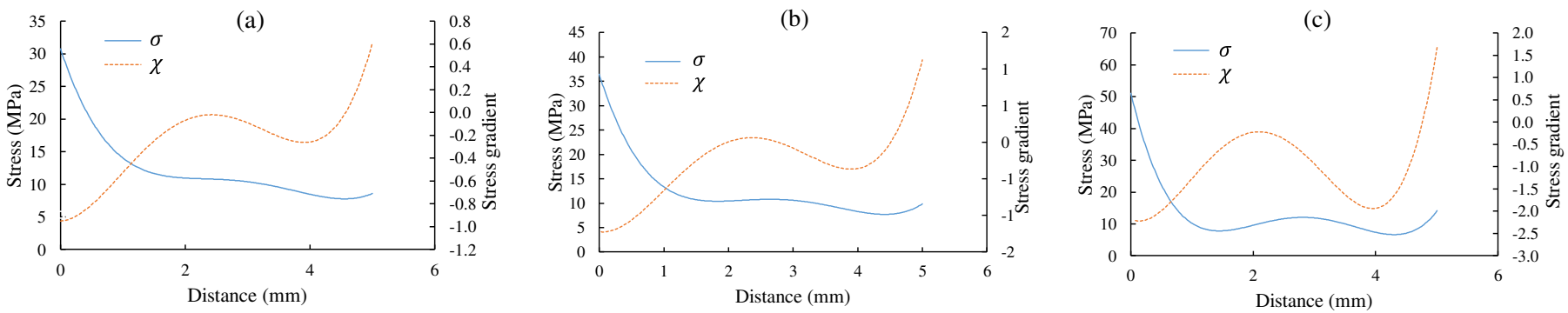

Fig. 8. Stress distributions and relative stress gradients for notched samples with $0^{\circ}$ raster angles at $\mathrm{S}=12.3$ MPa for (a) circular notched shape, (b) elliptical notch $(b / a=0.5)$, and (c) elliptical notch $(\mathbf{b} / \mathbf{a}=0.25)$.

It was found that the effective distances for the notched samples varied roughly between 0.047$0.052 \mathrm{~mm}$ for the samples with $0^{\circ}$ raster angle and $0.18-0.25 \mathrm{~mm}$ for the sample with $90^{\circ}$ raster orientations. The stress distributions and relative stress gradients as well as the effective distances were utilized to obtain the notch strength reduction factors for all notched samples and at all load levels. Figure 9 illustrates the notch strength reduction factor values versus the parameter $b / a$ for the samples with respectively $0^{\circ}$ and $90^{\circ}$ raster orientations.
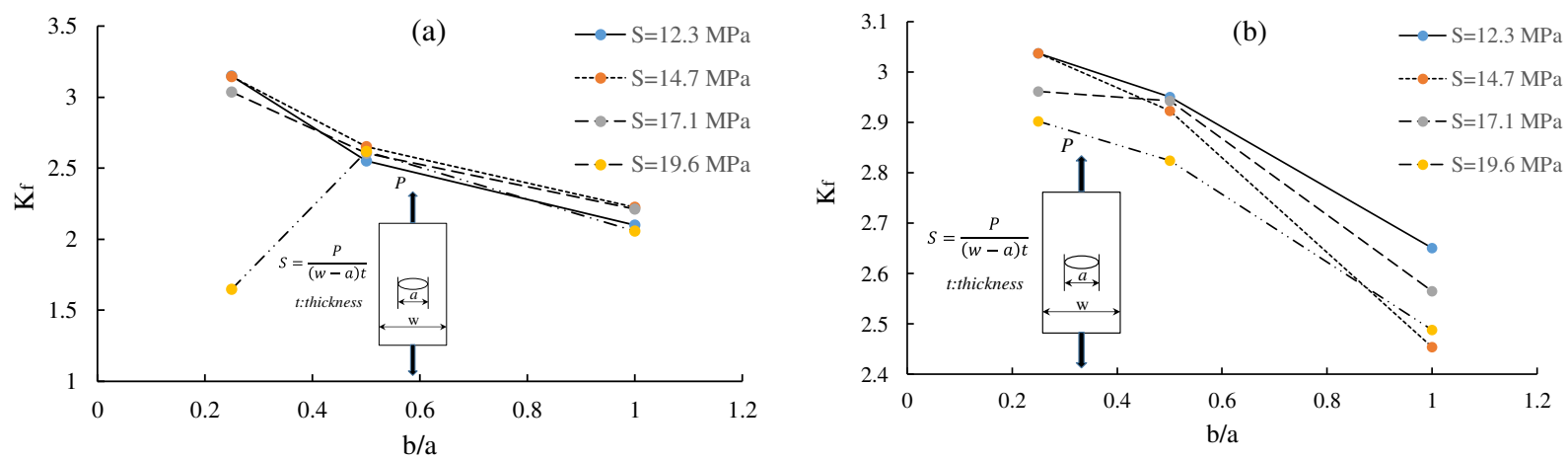

Fig. 9. Notch strength reduction factors versus parameter b/a for (a) $0^{\circ}$, and (b) $90^{\circ}$ raster orientations

As seen in Figure 9 and as expected, the notch strength reduction factors decrease with increasing notch tip radius. A drop in the value for the case of $0^{\circ}$ raster angle at $S=19.6 \mathrm{MPa}$ is due to the larger plastic strain component as compared to the other cases. Notch strength reduction factors can be used to predict the fatigue life of the notched samples as long as the smooth fatigue reference curve is known. Fatigue life predictions were fulfilled for the notched samples based on the available fatigue test data of the neat PLA material and cycle-dependent fatigue strength reduction factors. The predicted fatigue lives were compared with experimental fatigue test data, as 
evidenced in Figure 10. In this figure dashed lines indicate the upper and lower scatter bands with the factor of \pm 2 .

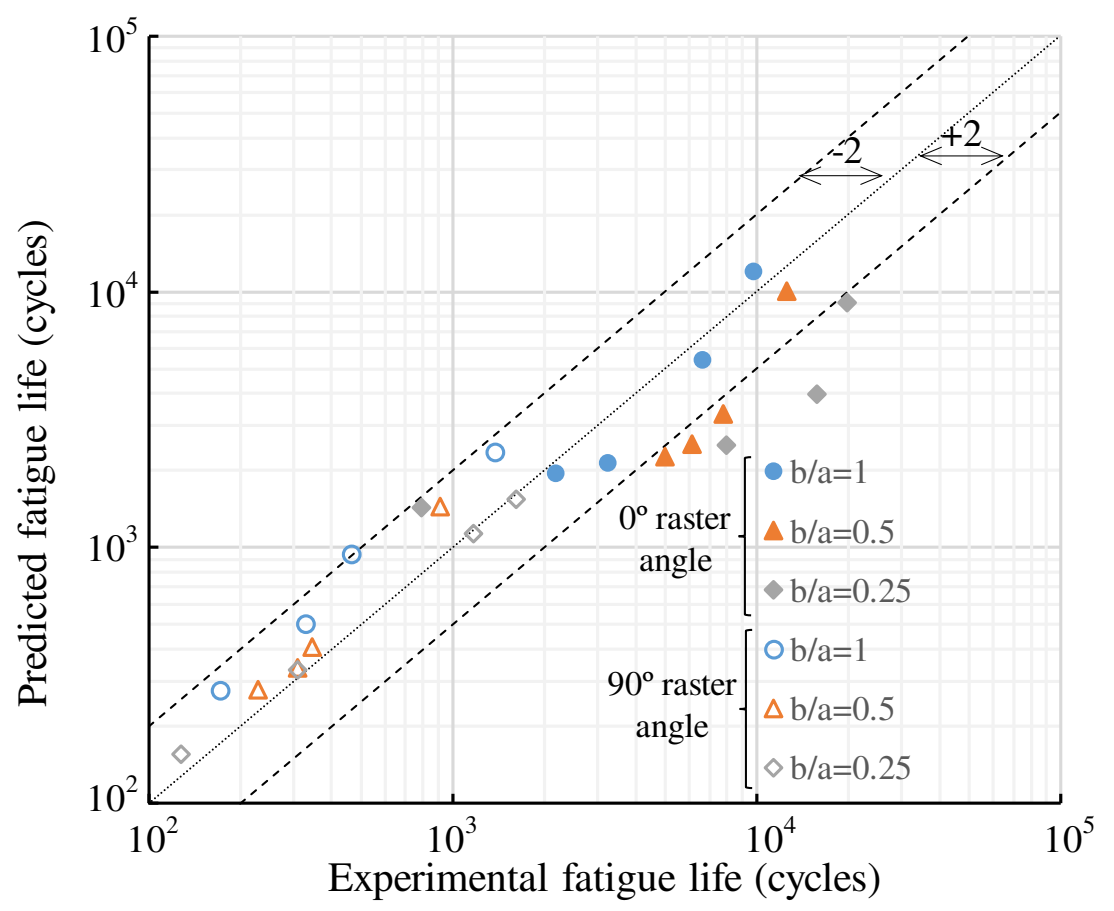

Fig. 10. Experimental and predicted fatigue life of the notched $A M$ samples at (a) $0^{\circ}$, and $(b) 90^{\circ}$ raster orientations

The majority of predicted life data through the use of volumetric approach fell within the factor of \pm 2 . This approach was efficient in estimating the fatigue life of samples with $90^{\circ}$ raster orientation having elliptical notch shape. A slight overestimation for the case of circular notched samples with $90^{\circ}$ raster angle is due to the effects of discontinuities between the filaments and stress concentrations in these areas. The impact of stress concentrations due to the rough surface for the samples with $0^{\circ}$ raster orientations was much less than that of the samples with $90^{\circ}$ raster angles. That is why the predicted life results through this approach showed slightly underestimation for the elliptical notched specimens with $0^{\circ}$ raster orientations. A relatively similar phenomenon can be found in ref. [25] in which rough surface of the un-notched as-built surface of AM metallic specimens acted as a local notch, resulting in decreased fatigue life.

\section{6- Conclusions}

Fatigue life of FDM-processed notched PLA samples were studied experimentally and numerically. The volumetric approach based on the theory of critical distance was utilized to obtain effective distances, fatigue notch factors, and fatigue lives. The effects of two different raster orientations were studied on the fatigue life taking into account the influence of voids between the filaments in the samples. Overall, the fatigue life predictions through use of volumetric approach 
were closely agreed with those obtained through experiments. It was observed that FDM-induced stress concentrations in the specimens acted similar to a notch, and were more noticeable for the samples with $90^{\circ}$ raster orientation as compared to those samples with $0^{\circ}$ raster angles.

Funding: This research was supported by Natural Sciences and Engineering Research Council of Canada (NSERC under grant RGPIN-217525). The authors are grateful for their support.

Conflict of interest: The authors declare no competing interests.

Data availability statement: The data presented in this study are available on request from the corresponding author.

Code availability: Not applicable.

Ethics approval: All professional ethics have been followed.

Consent to participate: Not applicable.

Consent for publication: Not applicable.

\section{References}

1. ZX Low, et al., Perspective on 3D printing of separation membranes and comparison to related unconventional fabrication techniques. Journal of Membrane Science, 2017. 523: p. 596-613.

2. I Larraza, et al., Cellulose and graphene based polyurethane nanocomposites for FDM 3D printing: Filament properties and printability. Polymers, 2021. 13(839).

3. JRC Dizon, et al., Mechanical characterization of 3D-printed polymers. Additive Manufacturing, 208. 20: p. 44-67.

4. L Zhao, et al., Development model and experimental characterization of residual stress of 3D printing PLA parts with porous structure. Applied Physics A, 2021. 127(98).

5. A Hernandez-Contreras, et al., Extended CT void analysis in FDM additive manufacturing components. Materials, 2020. 13(3831).

6. J Wang, et al., A novel approach to improvemechanical properties of parts fabricated by fused deposition modeling. Materials and Design, 2016. 105: p. 152-159.

7. SA Tronvoll, T Welo, and C. Elverum, The effects of voids on structural properties of fused deposition modelled parts: a probabilistic approach. The International Journal of Advanced Manufacturing Technology, 2018. 97: p. 3607-3618.

8. K Fayazbakhsh, M Movahedi, and J. Kalman, The impact of defects on tensile properties of 3D printed parts manufactured by fused filament fabrication. Materials Today Communications, 2019. 18: p. 140-148.

9. TW Kerekes, et al., Characterization of process-deformation/damage property relationship of fused deposition modeling (FDM) 3D-printed specimens. Additive Manufacturing, 2019. 25: p. 532544.

10. M Fischer and V. Schoppner, Fatigue Behavior of FDM Parts Manufactured with Ultem 9085. The Journal of The Minerals, Metals \& Materials Society, 2017. 69(3): p. 563-568.

11. $\mathrm{OH}$ Ezeh and L. Susmel, Fatigue strength of additively manufactured polylactide (PLA): effect of raster angle and non-zero mean stresses. International Journal of Fatigue 2019. 126: p. 319-326. 
12. S Ziemian, M Okwara, and C. Ziemian, Tensile and fatigue behavior of layered acrylonitrile butadiene styrene. Rapid Prototyping Journal, 2015. 21(3): p. 270-278.

13. MF Afrose, et al., Effects of part build orientations on fatigue behaviour of FDM-processed PLA material. Progress in Additive Manufacturing, 2016. 1: p. 21-28.

14. F Brenne and T. Niendorf, Effect of notches on the deformation behavior and damage evolution of additively manufactured $316 \mathrm{~L}$ specimens under uniaxial quasi-static and cyclic loading. International Journal of Fatigue, 2019. 127: p. 175-189.

15. G Nicoletto, et al., Influence of layer-wise fabrication and surface orientation on the notch fatigue behavior of as-built additively manufactured Ti6AI4V. International Journal of Fatigue 2020. 134: p. 1-10.

16. K S Chan and A. Peralta-Duran, A methodology for predicting surface crack nucleation in additively manufactured metallic components. Metallurgical and Materials Transactions A, 2019. 50A: p. 4378-4387.

17. M Kahlin, $\mathrm{H}$ Ansell, and J.J. Moverare, Fatigue behaviour of notched additive manufactured Ti6Al4V with as-built surfaces. International Journal of Fatigue 2017. 101: p. 51-60.

18. Nicoletto, G., Directional and notch effects on the fatigue behavior of as-built DMLS Ti6Al4V. International Journal of Fatigue 2018. 106: p. 124-131.

19. A A Ahmed and L. Susmel, A material length scale-based methodology to assess static strength of notched additively manufactured polylactide (PLA). Fatigue Fract Eng Mater Struct. , 2018. 41: p. 2071-2098.

20. A A Ahmed and L. Susmel, Static assessment of plain/notched polylactide (PLA) 3D-printed with different infill levels: Equivalent homogenised material concept and Theory of Critical Distances. Fatigue Fract Eng Mater Struct. , 2019. 42: p. 883-904.

21. C T Ng and L. Susmel, Notch static strength of additively manufactured acrylonitrile butadiene styrene (ABS). Additive Manufacturing 2020. 34: p. 1-15.

22. $\mathrm{O} \mathrm{H}$ Ezeh and L. Susmel, On the notch fatigue strength of additively manufactured polylactide (PLA). International Journal of Fatigue 2020. 136: p. 1-12.

23. K Solberg, D Wan, and F. Berto, Fatigue assessment of as-built and heat-treated Inconel 718 specimens produced by additive manufacturing including notch effects. Fatigue Fract Eng Mater Struct., 2020. 43: p. 2326-2336.

24. $\mathrm{K}$ Solberg and F. Berto, A diagram for capturing and predicting failure locations in notch geometries produced by additive manufacturing. International Journal of Fatigue 2020. 134: p. 16.

25. R Molaei and A. Fatemi, Fatigue performance of additive manufactured metals under variable amplitude service loading conditions including multiaxial stresses and notch effects: Experiments and modelling. International Journal of Fatigue 2021. 145: p. 1-15.

26. Dowling, N.E., Mechanical Behavior of Materials, Engineering methods for Deformation, Fracture, and Fatigue. Fourth Edition ed. 2013: Angshuman Chakraborty.

27. KN Smith, P Watson, and T. Topper, A stress strain function for the fatigue of metals. Journal of Engineering Materials and Technology 1970. 5(4): p. 767-778.

28. S. Hassanifard and S.M. Hashemi, On the strain-life fatigue parameters of additive manufactured plastic materials through fused filament fabrication process. Additive Manufacturing 2020. 32: p. 100973.

29. Neuber, H., Translation theory of notch stresses, Principles for exact stress calculations. 1958, US Office of Technical Services, Washington, DC, 1961: Springer.

30. Peterson, R.E., Notch sensitivity. In: Sines G, Waisman JL, editors. Metal Fatigue, 1959: p. 293306. 
31. Tanaka, K., Engineering formulae for fatigue strength reduction due to crack-like notches. International Journal of Fracture, 1983. 22: p. R39-R45.

32. Taylor, D., Geometrical effects in fatigue: a unifying theoretical model. International Journal of Fatigue, 1999. 21: p. 413-420.

33. Pluvinage, G., Fracture and fatigue emanating from stress concentrators. 2004: Kluwer Academic Publishers.

34. RD Averett, et al., The mechanical behavior of poly(lacticacid) unreinforced and nanocomposite films subjected to monotonic and fatigue loading conditions. Journal of Composite Materials, 2011. 45(26): p. 2717-2726. 